The Version of Scholarly Record of this Article is published in INTELLIGENCE (2015), available online at: http://dx.doi.org/10.1016/j.intell.2015.07.002. Note that this article may not exactly replicate the final version published in INTELLIGENCE.

Giofrè, D., \& Cornoldi, C. (2015). The structure of intelligence in children with specific learning disabilities is different as compared to typically development children. Intelligence, 52, 36-43. doi:10.1016/j.intell.2015.07.002

The structure of intelligence in children with specific learning disabilities is different as compared to typically development children

David Giofrè \& Cesare Cornoldi

${ }^{1}$ Department of General Psychology, University of Padua, Italy;

Correspondence concerning this article should be addressed to:

David Giofrè

Department of General Psychology

University of Padova

Via Venezia 8 - 35131 Padova, Italy

e-mail: david.giofre@gmail.com

Acknowledgement: we are extremely grateful to the colleagues who contributed to preparing the data file, and particularly to Drs. E. Stoppa, A.M. Antonucci, M. Frinco, M. Sironi, C. Miliozzi, E. Ramanzini, C. Vio, S. Santon, F. Bellelli, C. Bachmann, Ferioli, P., and the group of psychologists working at the LABDA - University of Padova. 


\begin{abstract}
Children with specific learning disabilities (SLDs) are characterized by a poor academic achievement despite an average intelligence. They are therefore typically assessed not only with achievement tests, but also with intelligence tests, usually the Wechsler Intelligence Scale for Children (WISC). The assumption of a discrepancy between IQ and achievement in children with SLD has been questioned, however, and the implications of using different measures in batteries of intellectual subtests have not been thoroughly investigated. The present study examined these issues, taking advantage of a large database of scores obtained in the ten core subtests of the WISCIV by a group of 910 Italian children with a clinical diagnosis of SLD, who were compared with the children considered for national standardization purposes. Our results support the doubts raised concerning the IQ-achievement discrepancy model, showing that relevant discrepancies can emerge even within the WISC profile. The four main WISC-IV indexes were found differently related to intelligence (measured by means of the $g$-factor) and the $g$ content of many subtests differed in children with SLD vis-à-vis typically-developing children. These results have important implications both theoretical, indicating that the $g$-factor is weakly identified in children with SLD children, and practical, indicating that the QI obtained with the WISC-IV may not be a good measure of intellectual functioning for children with SLD, which are discussed.
\end{abstract}

Keywords: Specific learning disorder; WISC-IV; General Ability Index; Full-Scale Intelligence Quotient; Cognitive profile of children with a SLD; Theories of intelligence

\title{
Highlights
}

- SLD children scores vary differently in the four WISC-IV indexes.

- The four IQ indexes are differently related to $g$.

- There are differences in the $g$ content of the indexes and many subtests between SLD cases and controls. 


\section{The structure of intelligence in children with specific learning disabilities is different as compared to typically development children}

Intelligence is one of the most important and most often assessed constructs in psychology (Gottfredson, 1997a). It has been demonstrated, for instance, that intelligence tests such as the WISC, the WAIS and the Raven progressive matrices are among the psychological tests most commonly used in Europe, taking first, second and fourth places, respectively (Evers et al., 2012). This may be due to the importance of intelligence, which is also confirmed by the fact that intelligence can predict important academic and occupational outcomes, and performance in everyday life (e.g., Deary, Strand, Smith, \& Fernandes, 2007; Gottfredson, 1997b; Schmidt \& Hunter, 2004).

The WISC, and particularly the WISC-IV (Wechsler, 2003), which is the latest version to become available in many countries, is one of most often used psychological tests. The popularity of the WISC-IV is probably due to the fact that it is relatively quick to administer and it measures important cognitive factors of relevance to the assessment of both typical populations and clinical groups (Prifitera, Saklofske, \& Weiss, 2008). The WISC-IV, as presented in the test manuals, is structured around four factors, considered among the main basic factors underlying intelligence: i) the verbal comprehension index, which measures verbal abilities such as comprehension and verbal reasoning; ii) the perceptual reasoning index, which measures abilities such as abstract problem solving and the non-verbal manipulation of materials; iii) the working memory index, which measures the capacity of retaining and manipulating verbal material for a short period of time; and iv) the processing speed index, which measures the ability to respond promptly and focus attention on a task. These four factors have been amply studied and are supported both statistically and clinically (Wechsler, 2004).

Alternative factorial structures of the WISC-IV have also been proposed, however, drawing from different theories of intelligence. In the light of the CHC (Cattell, Horn and 
Carroll) theory of intelligence, for example, it has been argued that a five-factor structure is plausible in the WISC-IV (Keith, Fine, Taub, Reynolds, \& Kranzler, 2006), which would include the four factors of the classical WISC-IV, but also distinguish between a fluid and a visual factor. This five-factor structure has been questioned for at least two reasons: i) the model fit is not very different from that of the classical structure, implying that the five-factor structure is not necessarily superior; and ii) the model has a perfect loading of the fluid reasoning factor on the general factor $(g)$, which makes one of the two factors redundant (see Styck \& Watkins, 2014). In addition, only the ten core subtests are administered in many settings (including the clinical one), as this is what is typically prescribed to estimate a child's full-scale intelligence quotient (FSIQ), and only having data available on ten subtests makes it impossible to refer to the five-factor structure of the WISC-IV. Discussions on these issues have been further complicated by the hypothesis that the structure of intelligence may differ in children with an atypical development, e.g., in children with specific learning disabilities (SLDs) (see for a discussion Styck \& Watkins, 2014).

The debate concerning the structure of intelligence and its assessment with the WISC scale has strong implications for children with SLD, who are characterized by impairments in academic domains (e.g., reading or math), despite a good or normal IQ. In fact, IQ has traditionally been assessed in children with SLD in order to identify this discrepancy between intelligence and academic achievement (Fuchs \& Fuchs, 2006). This approach has also been preserved in recent research not founded on the concept of discrepancy with a view to ruling out any intellectual disability that might explain SLD children's weaknesses in the academic domain (DSM-5; American Psychiatric Association, 2013).

In many countries, the WISC is the most popular test for assessing intelligence in children, especially in cases of SLD. The test, and the WISC-IV version in particular, not only affords a measure of general IQ, but also enables us to investigate different aspects of the performance of children with SLD. To give an example, recent studies using the ten core 
subtests and the classical four-factor structure found the performance of children with SLD strongly impaired in the Working Memory and, to a lesser extent, in the Processing Speed, but not in the Perceptual Reasoning or Verbal Comprehension indexes (Cornoldi, Giofrè, Orsini, \& Pezzuti, 2014; De Clercq-Quaegebeur et al., 2010; Poletti, 2014). Such evidence suggests that the intelligence of children with SLD may be organized differently from that of typicallydeveloping children, especially as regards the role of working memory (WM) and processing speed (PS).

Working memory and intelligence (as measured by the $g$-factor) are very closely correlated in typically-developing children, although they are separate constructs (Cornoldi \& Giofrè, 2014; Cornoldi, Orsini, Cianci, Giofrè, \& Pezzuti, 2013; Demetriou et al., 2014; Giofrè, Mammarella, \& Cornoldi, 2013). The fact that children with SLD may be of normal intelligence but impaired in their WM thus raises problems particularly for theories based on the assumption that WM and $g$ are hardly distinguishable and almost isomorphic (e.g., Martínez et al., 2011). The fact that PS seems to be impaired in children with SLD also contrasts with some theories of intelligence that hypothesize a fundamental role for processing speed in explaining intelligence, based on a large body of literature showing a relationship between intelligence and processing speed (for a review see Jensen, 2006)--though it has also been claimed that this relationship is not very strong (Hunt, 1980, 2011). In fact, the $g$-content of many processing speed tasks is relatively small, making it very hard to claim that differences in processing speed equate to differences in intelligence level.

Differences in WM, and especially in the processing speed, between normal and SLD groups therefore do not necessarily imply differences in the $g$-factor, and including them in a measure of intelligence can pose problems. In addition, impairments in cognitive processing abilities (which include both WM and processing speed) are often indicated as the core deficits in children with SLD (see Johnson, Humphrey, Mellard, Woods, \& Swanson, 2010), 
so impairments in processing speed may share a common cause with impairments in WM, and be unrelated to differences in intelligence.

Finally, although research provides only weak evidence of a deficit in the domain measured by the Verbal Comprehension Index in children with SLD, it is noteworthy that these children often have difficulties in the verbal domain. For example, many children with SLD had previously been diagnosed with an early language problem (American Psychiatric Association, 2013), and they may reveal verbal impairments in tasks associated with intellectual functioning. They often have trouble not only with word recognition and spelling, but also in reading comprehension (e.g., Swanson \& Ashbaker, 2000). Hence it can also hypothesized that weaknesses in the Verbal Comprehension Index do not necessarily coincide with weaknesses in $g$ (and therefore in intelligence).

To sum up, using a classical intelligence test like the WISC and assuming the generalizability of the theories of intelligence underlying such test may prove problematic in the case of children with SLD, and the matter warrants more in-depth investigation. In the present study we examined these issues by taking advantage of the availability of WISC-IV measures obtained for a large sample of children with SLD.

The first aim of the present study was to test whether children with SLD are impaired in the WISC-IV, and in which particular tasks/factors. In a first set of analyses, we expected to find that children with a diagnosis of SLD have specific deficits in some intellectual measures. In particular, we tried to confirm previous findings of moderate-to-severe impairments of children with SLD in both the Working Memory and the Processing Speed indexes, but not in the Verbal Comprehension or in the Processing Speed indexes (Cornoldi et al., 2014; Poletti, 2014). We therefore expected only small differences between the SLD and controls in the General Ability Index (GAI; Prifitera et al., 2008), which combines the Verbal Comprehension and the Perceptual Reasoning indexes, but moderate or large differences in 
the Cognitive Proficiency Index (CPI; Saklofske, Coalson, Raiford, \& Weiss, 2010), which combines the Working Memory and the Processing Speed indexes.

A second aim of the present study was to identify the best-fitting model of intelligence for children with SLD. As different models are theoretically plausible, we tested in our sample different solutions based on the WISC-IV looking for further evidence that the classical four-factor structure is tenable in children with SLD, contrasting it with alternative factor structures, that have also been associated to WISC, but derive from classical theories of intelligence, either based on a single factor (Spearman, 1904; see also Jensen, 1998) or on a verbal/spatial distinction (Vernon, 1950; see also Johnson and Bouchard, 2005). Having established the most appropriate solution, we tested whether any of the tasks revealed crossloadings on multiple factors due to the specific characteristics of children with SLD.

A third aim, connected to the first two, was to test whether the structure of intelligence derived from the WISC-IV is "equivalent" for typically-developing children and those with SLD. In fact, based on previous evidence on SLD and on the hypothesis that the four-factor structure was tenable in our sample, we assumed however that the relationship on the latent level between the four factors of the WISC-IV might differ between a group with SLD and a group of typically-developing children. In particular we hypothesized that the direct link between $g$ and some of the first-order factors should be lower in SLD than in typically developing children, and that the indirect effect of $g$ on the manifest variable (i.e., for the corresponding subtests) was lower in the SLD group than in the typically-developing children.

In fact, the relationship between the $g$-factor and the other factors of the WISC-IV might, in the case of SLD, be weaker in two domains (i.e., working memory, processing speed) and maybe also in a third domain (verbal comprehension), in which case the $g$-loading of the tasks used to measure these factors should also be lower in the SLD than in the typically-developing group. If so, differences emerging in tasks in the working memory, 
processing speed and verbal comprehension domains would reflect differences not necessarily in $g$, but in underlying factors. Finally, we also examined whether, due to the presence of specific deficits in the case of SLD, loadings of the tasks on their respective factors were the same, and whether the correlation between the first-order factors (i.e., the perceptual reasoning, verbal comprehension, working memory and processing speed) was weaker in the SLD than in the typically-developing group.

\section{Method}

\section{Participants}

Under the auspices of the Italian Association for Learning Disabilities (AIRIPA), we invited a group of experts to provide data obtained by administering the WISC-IV to children with a certified clinical diagnosis of learning disorder or intellectual disability, based on the ICD-10 International Coding System. A preliminary analysis was conducted when the first data arrived, and the results were published (Cornoldi et al., 2014), but none of the analyses discussed in this paper were the object of said previous report. The present analysis also concerns more than 600 new cases not considered in the previous study and excludes cases with a diagnosis of intellectual disability. For the present study, we collected information on 910 children and adolescents between 7 and 16 years of age who underwent a WISC-IV assessment on the 10 core subtests, and were diagnosed clinically with SLD ( $\mathrm{M}_{\mathrm{age}}=10.92$ $[\mathrm{SD}=2.41]$; females $=40 \%)^{1,2}$. Based on the classification of SLD adopted in Italy (the ICD10), the sample included: 216 cases of F81.0 (specific reading disorder); 54 cases of F81.1 (specific spelling disorder); 53 cases of F81.2 (specific disorder of arithmetical skills); 359 cases of F81.3 (mixed disorder of scholastic skills); 34 cases of F81.8 (other developmental disorders of scholastic skills); 12 cases of F81.9 (developmental disorder of scholastic skills, unspecified); and 182 children with two or more of the above diagnoses within the F81 
category. These children and adolescents were compared with a typically-developing sample whose data were drawn from the Italian WISC-IV manual (Orsini, Pezzuti, \& Picone, 2012).

\section{Instrument}

We used the recently-published Italian adaptation of the WISC-IV (Orsini et al., 2012) that retains the Full-Scale IQ and the four main indexes, and also includes the two additional indexes (General Ability and Cognitive Proficiency). Judging from the WISC-IV Italian testmanual, the internal consistencies, test-retest and inter-rater stability, and standard errors of measurement are comparable with those of the English version (Wechsler, 2003).

For the purposes of the present study, we examined the scores obtained in the 10 core subtests of the WISC-IV, i.e. Block Design (BD), Similarities (SI), Digit Span (DS), Picture Concepts (PCn), Coding (CD), Vocabulary (VC), Letter-Number Sequencing (LN), Matrix Reasoning (MR), Comprehension (CO), and Symbol Search (SS). We calculated the FullScale IQ (FSIQ) from the sum of the ten subtests, and the four main indexes, i.e.: the Perceptual Reasoning Index, which includes Block Design, Picture Concepts, and Matrix Reasoning; the Verbal Comprehension Index, which includes Similarities, Vocabulary, and Comprehension; the Working Memory Index, which includes Digit Span and Letter-Number Sequencing; and the Processing Speed Index, which includes Coding and Symbol Search. We then calculated the scores for the two additional indexes, i.e.: the General Ability, obtained from the Verbal Comprehension and the Perceptual Reasoning indexes; and the Cognitive Proficiency, obtained from the Working Memory and the Processing Speed indexes. Additional information on the subtests, main indexes and additional indexes is available elsewhere (Flanagan \& Kaufman, 2004; Wechsler, 2004).

\section{Data analysis plan}

The R program (version 3.1.2; R Core Team, 2014) with the "lavaan" library (version 0.5-17; Rosseel, 2012) was used for the confirmatory factor analysis (CFA) and multi-group 
CFA (MG-CFA). Model fit was assessed using various indexes according to the criteria suggested by Hu and Bentler (1999). We considered the chi-square $\left(\chi^{2}\right)$, the comparative fit index (CFI), the non-normed fit index (NNFI), the standardized root mean square residual (SRMR), and the root mean square error of approximation (REMSEA); the chi-square difference $\left(\Delta \chi^{2}\right)$, and the Akaike information criterion (AIC) were also used to compare the fit of alternative models (Kline, 2011). The assumption of multivariate normality and linearity was tested using the PRELIS with the LISREL 8.80 software (Jöreskog \& Sörbom, 2002, 2006).

\section{Results}

\section{Patterns of performance in children with SLD}

Table 1 shows correlations and mean standardized scores obtained by the SLD group in the WISC-IV subtests, main indexes and additional indexes. Taking for granted that the standardized mean scores of typically-developing children are, by definition, $100(\mathrm{SD}=15)$ in the case of the composite measures, and $10(\mathrm{SD}=3)$ in the case of specific subtests, it is immediately obvious that the standardized scores obtained by the children with SLD were average in the subtests associated with the General Ability Index and the underlying Verbal Comprehension and Perceptual Reasoning indexes, but lower than average in the other subtests.

Univariate skewness and kurtosis values were under 1.0. The measure of multivariate kurtosis was 1.05 , which is considered relatively small, so the estimation method that we chose (maximum likelihood) is robust against several types of violation of the multivariate normality assumption (Bollen, 1989).

\section{WISC-IV structure of cognitive abilities in children with SLD}

We ran a series of CFA in the SLD group, comparing different models. 
Model 1 investigated a single $g$-factor; it provided a poor fit with the data (Table 2). Model 2 investigated two distinct verbal (measured by Vocabulary, Similarities, Comprehension, Digit Span, and Letter-Number Sequencing) and non-verbal factors (measured by Block Design, Picture Concepts, Matrix Reasoning, Coding, and Symbol Search) and provided a poor fit with the data (Table 2).

Model 3 investigated the classical four-factor structure of the WISC-IV (four-factor model; Fig. 1); it provided a good fit with the data (Table 2). The correlation between the factors was only moderate $(M=.366 ; M d=.391)$. The residual correlation between Picture Concepts and Similarities was high (.11) (Kline, 2011), however, so we attempted to improve the model fit in subsequent models.

In Model 3b we allowed the residual Picture Concepts and Similarities to correlate: as shown in Table 2, the model fit improved significantly and proved more parsimonious (the AIC was lower) than in Model 3.

In Model 3c we allowed Picture Concepts to load on the Verbal Comprehension factor: as shown in Table 2, here again the model fit improved significantly and proved more parsimonious than in Model 3.

Model 4 investigated a hierarchical four-factor structure of the WISC-IV with a $g$ factor at the top and four first-order factors (Figure 1). Though theoretically feasible, this model did not reveal a better fit than Model 3 (Table 2). It is worth noting that the values for the path from $g$ to the verbal comprehension, perceptual reasoning, working memory, and perceptual reasoning indices were $.92, .63, .58$, and .31 , respectively, which were lower than in the control group in many cases (Figure 1), so we further investigated this finding using a multi-group CFA approach. 


\section{Multi-group CFA on SLD and typically-developing children on the WISC-IV first-order} structure.

We initially compared the SLD and the typically-developing samples with a multigroup approach using Model 3 (i.e., without a $g$-factor at the top). When we tested the structural equivalence of the two groups (Model 5), the model showed a good fit, confirming that the same structure holds in both groups.

In the next analysis (Model 6), we tested the equivalence of factor loadings $\left(\mathrm{H}_{\Lambda}\right)$ for the two groups. The chi-square difference test indicated that Model 6 was not statistically superior to Model 5, the fit indices differed little from the previous model, and there were no residuals greater than .10 (see Kline 2010 on this issue), so we went a step further to test other forms of invariance.

In Model 7, we tested the invariance of latent variances and covariances. As shown in Table 2, the fit was poor, becoming worse than in the previous models. The chi-square difference test strongly suggested that this model should be rejected (Table 2). The SLD group also included 12 absolute correlation residuals $>.10$. Taken together, these results meant that this model should be rejected (see Kline, 2011).

\section{Multi-group CFA on SLD and typically-developing children on the WISC-IV first-order structure}

Based on the results of our previous analyses (Models 6 and 7), we added a secondorder factor structure (with a $g$-factor at the top) in Model 8, and factor loadings at the firstorder level (from the manifest variables to the first-order factors) were constrained to make them equal across groups (consistently with Model 6). The model provided a good fit with the data, so we tested the invariance at the second-order factor level. 
In Model 9 the measurement error of the Perceptual Reasoning Index and the direct link from $g$ to Perceptual Reasoning Index were set to be invariant in the two groups. The model did not differ statistically from the previous one, showing that the link from $g$ to Perceptual Reasoning Index and the error variance in Perceptual Reasoning Index can be considered group-invariant. As Kline recommended (2011), to fit this model we set the Verbal Comprehension Index as the reference variable. The next model (Model 9b) was essentially the same as Model 9 except for the fact that we used the Perceptual Reasoning Index as the reference variable (Table 2). This model was taken for reference and used to compare all the subsequent models.

In Models 10, 11 and 12, we set the following to be group-invariant, one at a time: i) the direct link from $g$ to single first-order factors; and ii) the corresponding error variance of the specific first-order factors (i.e., the error variance of the Verbal Comprehension, Working Memory, and Processing Speed, respectively). In Model 10 the Verbal Comprehension Index was not group-invariant (Table 2); in Model 11 the Working Memory Index was not groupinvariant either (Table 2); and in Model 12, nor was the Processing Speed Index. In this last case, the results were less clear, however: the chi-square difference was significant, all the fit indices were similar, and the AIC was close to that of the reference model (Model 9b).

To sum up, judging from our results, only the Perceptual Reasoning loading on $g$ (and its error variance) seems to be group-invariant, while that of the Working Memory, Verbal Comprehension and Processing Speed indexes are not

\section{Loadings on $g$ and variances for each subtest}

Since many second-order loadings on $g$ were not group-invariant (i.e., Verbal Comprehension, Working Memory and Processing Speed indexes), we investigated $g$ loadings and $g$-variances for each task. We therefore fitted two separate analyses for the SLD and the typically-developing group using a hierarchical four-factor structure (Model 4). The 
parameters estimated are shown in Figure 1. We estimated the indirect influence (or $g$ content) of the second-order factor $(g)$ on the manifest variables (each subtest of the WISCIV) in terms of loadings and variances. As shown in Table 3, the $g$-content was considerably lower in the SLD group compared to controls, particularly for working memory tasks, but also for Verbal Comprehension and Processing Speed tasks. There seemed to be a difference of about $14-15 \%$ in terms of $g$-variance in the SLD group (for WM tasks). For the Verbal Comprehension, we found a difference of about $8-10 \%$ in terms of $g$-variance, while for the Processing Speed the loadings on $g$ were weak in both groups, but the proportion of variance explained by $g$ in the SLD group was very weak (about $2 \%$, for the Coding subtest).

To sum up: i) the performance of children with SLD is impaired in the working memory and in the processing speed; ii) the structure of these children's intelligence is consistent with four factors, but the Picture Concepts in the SLD group has a residual correlation on Similarities with a significant loading on the Verbal Comprehension Index, suggesting that it may demand some form of verbal strategy (see Models $3 b$ and 3c); iii) only the Perceptual Reasoning Index had the same loadings on the g-factors in the SLD and typically-developing children, showing that many tasks may assess intelligence differently in the two groups.

\section{Discussion}

This study examined the intellectual performance of children with SLD as identified by the WISC-IV, and the similarities and differences between how the intelligence of typically-developing and SLD children is organized. Our results are therefore limited to the case of the WISC-IV and other intelligence measures need to be considered before these findings can be generalized. It seemed crucial to refer initially to the WISC-IV battery, however, for three main reasons: (i) the WISC scales are the most widely-used procedures for assessing intelligence in many countries (Evers et al., 2012); (ii) the WISC-IV makes it 
possible to distinguish between scores on different indexes, which seem to be affected to a different degree in children with SLD; and (iii) the WISC-IV is the intelligence test typically used in the diagnosis of SLD, and this makes it possible to collect large datasets.

First of all, our findings in a large sample of cases support the evidence of children with a diagnosis of SLD typically having average scores in measures of verbal and non-verbal intelligence but lower scores in measures of working memory and processing speed. Our results also confirm the observation that the strong relationship between the latter two cognitive components and intelligence applies to a lower extent in children with SLD (Cornoldi et al., 2014; Poletti, 2014). Some differences in the patterns of performance reported elsewhere in children with SLD (e.g., De Clercq-Quaegebeur et al., 2010) may be due to the small size or specific characteristics of the samples considered. Our results have theoretical and clinical implications because they confirm that SLD children may have a normal level of intelligence (as measured by the General Ability Index), despite a poor performance in the Working Memory and Processing Speed indexes.

The second aim of our study was to test the WISC-IV structure in children with SLD. We compared different models derivable from the WISC-IV measures to pinpoint the best description of how intelligence is organized in children with SLD. We confirmed that the WISC-IV has a four-factor structure in children with SLD too, but one task (Picture Concepts) had loadings on two factors, showing that children with SLD may also use verbal strategies to solve non-verbal problems. It is also worth mentioning that the four factor were not found strongly inter-correlated in this group.

Concerning our third aim, we tested the equivalence of the WISC-IV in typicallydeveloping children and those with SLD. The results showed that the loading on $g$ (i.e., the influence of $g$ on the first-order factors) of three of the four factors (the Working Memory, Verbal Comprehension, and Processing Speed) was lower in the SLD children than in the typically-developing group. This finding may have important consequences: if the $g$-content 
of most of the subtests (i.e., the indirect influence of $g$ on the manifest variables) can depend on the g-loading of each factor, and if it differs (as we found in three out of four factors), then the subtests may have different $g$-loadings, meaning that they are not measuring intelligence (the $g$-factor) in the same way. Differences between the two groups in subtests with different $g$-loadings therefore do not necessarily coincide with differences in intelligence. This kind of "bias" seems to be particularly relevant for the WM measures since the $g$-content of Digit Span and Symbol Search was considerably lower in the SLD group.

The $g$-content of the tasks in the Processing Speed Index seemed to be lower too. We found the loading of the Processing Speed Index on $g$ statistically lower in the SLD than in the typically-developing group. Processing speed tasks (i.e., the Coding and the Symbol Search) may demand an involvement of the executive attention component, which seems to be impaired in children with SLD (Johnson et al., 2010). Deficits in the processing speed component and in the WM may therefore share the same basic mechanisms and be due to the same executive attention problem -- confirming, here again, that differences in tasks measuring processing speed may not reflect differences in $g$. Importantly, this situation is probably not limited to SLD, but could well apply to other groups of children with impairments in executive processes, such as those with ADHD (Cornoldi, Giofrè, Calgaro, \& Stupiggia, 2013). Like those with SLD, children with ADHD typically score lower in the Processing Speed Index, and a deficit in the Coding task, for example, is very common in such children (Calhoun \& Mayes, 2005). But when children with ADHD are asked only to copy coding symbols (a task assumed to demand a lesser degree of attentional control), their performance does not differ statistically from that of controls (Jacobson et al., 2011). Once again, differences in Processing Speed Index scores may not necessarily coincide with differences in the $g$-factor, partly because the $g$-content is very limited in children who have executive problems, as is the case in children with SLD. We believe that processing speed is a useful index when testing deficits in processing speed in SLD (or ADHD), and should be 
measured, but it should not be included in the calculation of intelligence because it is a very weak measure of this construct, in atypical groups at least.

Based on our findings the General Ability Index - which only includes the Perceptual Reasoning and the Verbal Comprehension indexes - seems to be a valid measure of IQ in children with SLD. Some authors have provided guidelines on the General Ability Index, in terms of criteria for describing the discrepancy between this index and the Full Scale IQ. Flanagan and Kaufman (2004) suggested that, when the difference between the highest and the lowest indexes in the WISC-IV is greater than 23 points, then the Full Scale IQ should not be adopted, and other indices (e.g., the General Ability Index) should be considered instead. This approach was recently questioned by other authors, however, who argued that using this procedure about $40 \%$ and $50 \%$ of Full Scale IQs (in the USA and Italy, respectively) would be dismissed (Orsini, Pezzuti, \& Hulbert, 2014), and they proposed a different discrepancy criterion (e.g., 40 points) (Orsini \& Pezzuti, 2014). In our opinion, every criterion (be it 40 or 23 points) is largely arbitrary and the Full Scale IQ should be dismissed and the General Ability Index preferred for all children with SLD because differences in the Working Memory and Processing Speed indexes may not necessarily reflect differences in intelligence, so the estimated Full Scale IQ may be somewhat biased.

Even the General Ability Index may sometimes pose problems as a measure of intelligence in children with SLD. In the present study, we found no large differences in the mean scores obtained on the Verbal Comprehension Index by typically-developing controls and children with SLD (Cornoldi et al., 2014). On the other hand, a large body of literature indicates that children with SLD often have trouble in tasks involving verbal reasoning or the retrieval of verbal information (Swanson \& Ashbaker, 2000; Swanson, 1999). Children with SLD may often have a history of language impairments (see the DSM-V; American Psychiatric Association, 2013), and children with language impairments often have deficits in the Verbal Comprehension Index (Wechsler, 2004). Our sample of children with SLD 
revealed no deficit in the Verbal Comprehension Index, but we also found the link from $g$ to the Verbal Comprehension Index weaker, and the $g$-content of the verbal comprehension tasks lower in these children. This means that, in many cases, differences in the Verbal Comprehension Index may not coincide with differences in level of intelligence. That is why we believe that, when children with SLD also have associated language problems, even the value of the General Ability Index becomes questionable; the Perceptual Reasoning Index could be a valid alternative in such cases.

Among the four main indices in the WISC-IV, only the Perceptual Reasoning Index seemed to have the same loading on the $g$-factor in children with typical development and children with SLD. Not all the Perceptual Reasoning Index tasks seemed to work in the same way in the SLD and typically-developing groups, however. Among the various perceptual reasoning tasks, Matrix Reasoning seemed to be both highly $g$-loaded and invariant in the two groups, so using tasks that involve matrix reasoning - such as the matrix reasoning or Raven progressive matrices (e.g., Raven \& Court, 1998) - may make sense in the case of children with SLD. Conversely, tasks such the Picture Concepts may additionally demand some sort of verbal reasoning for the SLD group (but not for controls, as shown by Keith et al., 2006), so it is hard to say whether they provide a measure of the $g$-factor or of the use of some other strategies. This observation seems to be confirmed by our finding that the $g$-content of the Picture Concepts was lower in the SLD group than in the control group. Although the Perceptual Reasoning Index does not seem to be biased in children with SLD, it is worth mentioning that the reliability at levels of the indices is often quite poor (Watkins \& Smith, 2013). A complete non-verbal estimation of IQ using other instruments may therefore often be more appropriate in children with SLD.

Despite its important theoretical and clinical implications, this study has a number of limitations that should be addressed in future research. For a start, we only had data on the 10 core subtests of the WISC-IV; other subtests could be used too. Second, we only had data on 
the WISC-IV; other, recently-released tests could be used instead, such as the WISC-V (Wechsler, 2014), or the Leiter-3 (Roid, Miller, Pomplun, \& Koch, 2013). Indeed, it would be very interesting to see how children with SLD perform with the WISC-V for a number of reasons. For instance, the tasks in the WISC-V no longer include Picture Concepts as a primary measure of fluid intelligence, meaning that the estimate of fluid reasoning may be less biased than in the WISC-IV; and the General Ability Index comprises 5 tasks (3 nonverbal, and only 2 verbal), thus allowing for the fact that children with SLD may have problems in the verbal domain. Similarly, by excluding both verbal and processing subtests from the calculation of IQ, the Leiter-3 could provide a better estimate of the intellectual abilities of children with SLD.

Future research is also needed to examine the intellectual patterns of other atypical groups, and especially children with ADHD, who seem to be similar in some ways to cases of SLD (Goker, Uneri, Guney, Dinc, \& Hekim-bozkurt, 2014).

In conclusion, despite some limitations, the present study offers important information on the generalizability of current descriptions of intelligence derived by the use of intelligence tests. Our results showed that children with SLD and typically-developing controls have partly similar and partly different intellectual patterns, as measured by the WISC-IV. This scale proved useful, but not all the indexes and subtests seem to have the same $g$-content in the two groups. This finding suggest that the core components of intelligence might be different in children with SLD compared to controls. In fact, only the Perceptual Reasoning Index, with two subtests in particular, was found both to have high $g$-loadings and with no evidence of biases between the two groups. This has also implications for the assessment, showing that, when assessing intellectual abilities in children with SLD, it therefore seems reasonable to prefer a less biased measure, such as the General Ability Index (Cornoldi et al., 2014; Poletti, 2014), or other non-verbal measures of intelligence. Results have also important theoretical implications, because they confirm that children with SLD can be of average 
intelligence even though they obtain lower estimates of overall IQ. In fact, children with SLD struggle with working memory and processing speed tasks and this can considerably lower their IQ estimates, even though these factors are weakly related to the $g$-factor compared to typical developing children. Such evidence further confirms that intelligence and other basic aspects (e.g., working memory or processing speed) may be very highly related in the normal population but not necessarily in other groups, suggesting that these constructs should be considered separable. Our results can also have clinical implications, indicating that it may be very difficult to assess intelligence in children with atypical development, so examiners will need to use their own "intelligence" when interpreting the results of such scales. 


\section{References}

American Psychiatric Association. (2013). Diagnostic and Statistical Manual of Mental Disorders (5th ed.). Arlington, VA: American Psychiatric Publishing.

Bollen, K. A. (1989). Structural Equations with Latent Variables. New York, NY: Wiley.

Calhoun, S. L., \& Mayes, S. D. (2005). Processing speed in children with clinical disorders. Psychology in the Schools, 42(4), 333-343.

Cornoldi, C., \& Giofrè, D. (2014). The crucial role of working memory in intellectual functioning. European Psychologist, 19(4), 260-268.

Cornoldi, C., Giofrè, D., Calgaro, G., \& Stupiggia, C. (2013). Attentional WM is not necessarily specifically related with fluid intelligence: The case of smart children with ADHD symptoms. Psychological Research, 77(4), 508-515.

Cornoldi, C., Giofrè, D., Orsini, A., \& Pezzuti, L. (2014). Differences in the intellectual profile of children with intellectual vs. learning disability. Research in Developmental Disabilities, 35(9), 2224-2230.

Cornoldi, C., Orsini, A., Cianci, L., Giofrè, D., \& Pezzuti, L. (2013). Intelligence and working memory control: Evidence from the WISC-IV administration to Italian children. Learning and Individual Differences, 26, 9-14.

De Clercq-Quaegebeur, M., Casalis, S., Lemaitre, M.-P., Bourgois, B., Getto, M., \& Vallée, L. (2010). Neuropsychological profile on the WISC-IV of French children with dyslexia. Journal of Learning Disabilities, 43(6), 563-74.

Deary, I. J., Strand, S., Smith, P., \& Fernandes, C. (2007). Intelligence and educational achievement. Intelligence, 35(1), 13-21.

Demetriou, A., Spanoudis, G., Shayer, M., van der Ven, S., Brydges, C. R., Kroesbergen, E., ... Swanson, H. L. (2014). Relations between speed, working memory, and intelligence from preschool to adulthood: Structural equation modeling of 14 studies. Intelligence, 46, 107-121.

Evers, A., Muñiz, J., Bartram, D., Boben, D., Egeland, J., Fernández-Hermida, J. R., ... Urbánek, T. (2012). Testing practices in the 21st century. European Psychologist, 17(4), 300-319.

Flanagan, D. P., \& Kaufman, S. (2004). Essentials of Assessment with WISC-IV. New York, NY: Wiley.

Fuchs, D., \& Fuchs, L. S. (2006). Introduction to response to intervention: What, why, and how valid is it? Reading Research Quarterly, 41(1), 93-99.

Giofrè, D., Mammarella, I. C., \& Cornoldi, C. (2013). The structure of working memory and how it relates to intelligence in children. Intelligence, 41(5), 396-406.

Goker, Z., Uneri, O. S., Guney, E., Dinc, G., \& Hekim-bozkurt, O. (2014). Clinical and sociodemographic features of children and adolescents with specific learning disorder (SLD). American Journal of Psychiatry and Neuroscience, 2(6), 90-95.

Gottfredson, L. (1997a). Mainstream science on intelligence: An editorial with 52 signatories, history, and bibliography. Intelligence, 24(1), 13-23.

Gottfredson, L. (1997b). Why g matters: The complexity of everyday life. Intelligence, 24(1), 79-132.

Hu, L., \& Bentler, P. M. (1999). Cutoff criteria for fit indexes in covariance structure analysis: Conventional criteria versus new alternatives. Structural Equation Modeling, $6(1), 1-55$. 
Hunt, E. (1980). Intelligence as an information-processing concept. British Journal of Psychology, 71(4), 449-474.

Hunt, E. (2011). Human Intelligence. New York, NY: Cambridge University Press.

Jacobson, L. A., Ryan, M., Martin, R. B., Ewen, J., Mostofsky, S. H., Denckla, M. B., \& Mahone, E. M. (2011). Working memory influences processing speed and reading fluency in ADHD. Child Neuropsychology, 17(3), 209-224.

Jensen, A. R. (1998). The g factor: The science of mental ability. Westport, CT: Praeger.

Jensen, A. R. (2006). Clocking the Mind: Mental Chronometry and Individual Differences. Amsterdam: Elsevier.

Johnson, E., Humphrey, M., Mellard, D., Woods, K., \& Swanson, H. L. (2010). Cognitive processing deficits and students with specific learning disabilities: A selective metaanalysis of the literature. Learning Disability Quarterly, 33(1), 3-18.

Johnson, W., \& Bouchard, T. J. (2005). The structure of human intelligence: It is verbal, perceptual, and image rotation (VPR), not fluid and crystallized. Intelligence, 33(4), 393-416.

Jöreskog, K. G., \& Sörbom, D. (2002). PRELIS 2 User's Reference Guide (3rd ed.). Lincolnwood, IL: Scientific Software International.

Jöreskog, K. G., \& Sörbom, D. (2006). LISREL for Windows [Computer software]. Lincolnwood, IL: Scientific Software International.

Keith, T. Z. T., Fine, J. G. J., Taub, G. G. E., Reynolds, M. R., \& Kranzler, J. H. (2006). Higher-order, multisample, confirmatory factor analysis of the Wechsler Intelligence Scale for Children - fourth edition: What does it measure? School Psychology Review, 35(1), 108-127.

Kline, R. B. (2011). Principles and Practice of Structural Equation Modeling (3rd ed.). New York, NY: Guilford Press.

Martínez, K., Burgaleta, M., Román, F. J., Escorial, S., Shih, P. C., Quiroga, M. Á., \& Colom, R. (2011). Can fluid intelligence be reduced to "simple" short-term storage? Intelligence, 39(6), 473-480.

Orsini, A., \& Pezzuti, L. (2014). L' indice di abilità generale della scala WISC-IV [The WISC-IV General Ability Index]. Psicologia Clinica Dello Sviluppo, 18(2), 301-310.

Orsini, A., Pezzuti, L., \& Hulbert, S. (2014). The unitary ability of IQ in the WISC-IV and its computation. Personality and Individual Differences, 69, 173-175.

Orsini, A., Pezzuti, L., \& Picone, L. (2012). WISC-IV: Contributo alla taratura Italiana. [WISC-IV Italian Edition]. Florence, Italy: Giunti O. S.

Poletti, M. (2014). WISC-IV intellectual profiles in Italian children with specific learning disorder and related impairments in reading, written expression, and mathematics. Journal of Learning Disabilities.

Prifitera, A., Saklofske, D. H., \& Weiss, L. G. (2008). WISC-IV Clinical Assessment and Intervention (2nd ed.). Amsterdam: Elsevier.

R Core Team. (2014). R: A language and environment for statistical computing. Vienna, Austria: R Foundation for Statistical Computing.

Raven, J. C., \& Court, J. H. (1998). Raven Manual: Standard Progressive Matrices. Oxford, UK: Oxford Psychologist Press.

Roid, G. H., Miller, L. J., Pomplun, M., \& Koch, C. (2013). Leiter International Performance 
Scale-third edition. Los Angeles, CA: Western Psychological Services.

Rosseel, Y. (2012). lavaan: An R package for structural equation modeling. Journal of Statistical Software, 48(2), 1-36.

Saklofske, D. H., Coalson, D. L., Raiford, S. E., \& Weiss, L. G. (2010). Cognitive proficiency index for the Canadian edition of the Wechsler Intelligence Scale for Children - fourth edition. Canadian Journal of School Psychology, 25(3), 277-286.

Schmidt, F. L., \& Hunter, J. (2004). General mental ability in the world of work: Occupational attainment and job performance. Journal of Personality and Social Psychology, 86(1), 162-73.

Spearman, C. (1904). "General Intelligence," objectively determined and measured. The American Journal of Psychology, 15(2), 201.

Styck, K. M., \& Watkins, M. W. (2014). Structural Validity of the WISC-IV for Students With Learning Disabilities. Journal of Learning Disabilities.

Swanson, H. L. (1999). Reading comprehension and working memory in learning-disabled readers: Is the phonological loop more important than the executive system? Journal of Experimental Child Psychology, 72(1), 1-31.

Swanson, H. L., \& Ashbaker, M. H. (2000). Working memory, short-term memory, speech rate, word recognition and reading comprehension in learning disabled readers: Does the executive system have a role? Intelligence, 28(1), 1-30.

Vernon, P. E. (1950). The Structure of Human Abilities. London: Methuen.

Watkins, M. W., \& Smith, L. G. (2013). Long-term stability of the Wechsler Intelligence Scale for Children - fourth edition. Psychological Assessment, 25(2), 477-83.

Wechsler, D. (2003). WISC-IV Technical and Interpretive Manual. San Antonio, TX: The Psychological Association.

Wechsler, D. (2004). The Wechsler Intelligence Scale for Children - fourth edition. London, UK: Pearson Assessment.

Wechsler, D. (2014). Wechsler Intelligence Scale for Children - fifth edition. London, UK: Pearson Assessment. 


\section{Footnotes}

${ }^{1}$ Only children with a clinical diagnosis of SLD and data on their WISC-IV assessment (on at least 10 core subtests) were considered in this paper. In all, 36 children were excluded from the analysis for various reasons. In particular, we excluded children with missing or unreliable data $(\mathrm{n}=20)$, under or over the WISC-IV age range $(\mathrm{n}=4)$, with an IQ lower than $65(n=8)$, or neurological disorders $(n=4)$.

${ }^{2}$ Data were unavailable on the gender of 68 children. 
Table 1

Correlations, means (M), and standard deviations (SD), for children with SLD tested on the WISC-4

\begin{tabular}{|c|c|c|c|c|c|c|c|c|c|c|c|c|c|c|c|c|c|}
\hline & 1 & 2 & 3 & 4 & 5 & 6 & 7 & 8 & 9 & 10 & 11 & 12 & 13 & 14 & 15 & 16 & 17 \\
\hline \multicolumn{18}{|l|}{ Main index } \\
\hline 1.Verbal Comprehension & 1 & & & & & & & & & & & & & & & & \\
\hline 3.Working Memory & .285 & .278 & 1 & & & & & & & & & & & & & & \\
\hline 4.Processing Speed & .056 & .212 & .150 & 1 & & & & & & & & & & & & & \\
\hline 5.Full Scale IQ & .767 & .760 & .569 & .456 & 1 & & & & & & & & & & & & \\
\hline 6.General Ability & .863 & .806 & .335 & .151 & .905 & 1 & & & & & & & & & & & \\
\hline 7.Cognitive Proficiency & .224 & .323 & .711 & .770 & .673 & .322 & 1 & & & & & & & & & & \\
\hline \multicolumn{18}{|l|}{$\underline{\text { Subtest }}$} \\
\hline 8. Similarities & .813 & .438 & .224 & .034 & .661 & .761 & .174 & 1 & & & & & & & & & \\
\hline 9. Vocabulary & .847 & .339 & .245 & .043 & .646 & .729 & .189 & .578 & 1 & & & & & & & & \\
\hline 14. Digit Span & .213 & .182 & .799 & .071 & .423 & .236 & .555 & .173 & .184 & .173 & .111 & .117 & .180 & 1 & & & \\
\hline 15. Letter-Number Sequencing & .265 & .281 & .817 & .151 & .513 & .327 & .626 & .209 & .230 & .222 & .184 & .198 & .262 & .358 & 1 & & \\
\hline 16. Coding & .019 & .111 & .113 & .826 & .334 & .074 & .668 & -.023 & .036 & .030 & .059 & .073 & .124 & .063 & .118 & 1 & \\
\hline 17. Symbol Search & .095 & .252 & .157 & .839 & .451 & .198 & .683 & .100 & .052 & .078 & .189 & .152 & .229 & .067 & .159 & .453 & 1 \\
\hline $\mathrm{M}$ & 102.08 & 103.81 & 89.21 & 91.00 & 97.27 & 103.22 & 88.46 & 10.08 & 10.03 & 10.92 & 10.28 & 10.98 & 10.56 & 8.04 & 8.34 & 8.28 & 9.17 \\
\hline SD & 15.84 & 14.55 & 13.02 & 14.21 & 12.90 & 14.39 & 12.82 & 3.04 & 3.05 & 3.41 & 2.85 & 3.02 & 3.07 & 2.52 & 2.62 & 2.86 & 2.84 \\
\hline
\end{tabular}

Note. All coefficients $\geq .065$ are significant at .05 level. 
Table 2

Fit indices for different CFA and MG-CFA on SLD and typically-developing groups

\begin{tabular}{|c|c|c|c|c|c|c|c|c|}
\hline & $\chi^{2}(d f)$ & $\Delta \chi^{2}(\Delta d f)^{(\mathrm{mc})}$ & $p$ & RMSEA & SRMR & $C F I$ & $N N F I$ & $A I C$ \\
\hline \multicolumn{9}{|l|}{ CFA on SLD } \\
\hline (1) Single $g$ factor & $559.25(35)$ & -- & & .128 & .093 & .703 & .618 & 44096 \\
\hline (2) Modality dependent & $387.91(34)$ & -- & & .107 & .079 & .800 & .735 & 43927 \\
\hline (3) Four-factor model & $81.33(29)$ & -- & & .045 & .031 & .970 & .954 & 43630 \\
\hline$(3 b)$ & $68.55(28)$ & $12.78(1)^{(3)}$ & .000 & .040 & .028 & .977 & .963 & 43619 \\
\hline (3c) & $70.50(28)$ & $10.58(1)^{(3)}$ & .001 & .041 & .027 & .976 & .961 & 43621 \\
\hline (4) High-order structure & $99.10(31)$ & $17.77(2)^{(3)}$ & .000 & .049 & .039 & .961 & .944 & 43643 \\
\hline \multicolumn{9}{|l|}{ Multi-group CFA } \\
\hline \multicolumn{9}{|c|}{ Based on the four-factor model (Model 3) } \\
\hline (5) & $242.27(58)$ & -- & -- & .045 & .028 & .976 & .963 & 148517 \\
\hline (6) & $255.89(64)$ & $13.62(6)^{(5)}$ & .034 & .044 & .030 & .975 & .965 & 148519 \\
\hline (7) & $329.74(74)$ & $73.85(10)^{(6)}$ & .000 & .047 & .058 & .967 & .960 & 148572 \\
\hline \multicolumn{9}{|c|}{ Based on the high-order structure (Model 4) } \\
\hline (8) & 283.93(68) & -- & -- & .045 & .033 & .972 & .963 & 148539 \\
\hline (9) & $286.92(70)$ & $2.99(2)^{(8)}$ & .224 & .045 & .033 & .972 & .964 & 148538 \\
\hline$(9 b)$ & 283.93(69) & $0.00(1)^{(8)}$ & .951 & .045 & .033 & .972 & .964 & 148537 \\
\hline (10) & $299.86(71)$ & $15.92(2)^{(9 b)}$ & .000 & .046 & .034 & .971 & .963 & 148549 \\
\hline (11) & $303.26(71)$ & $19.33(2)^{(9 b)}$ & .000 & .045 & .037 & .970 & .962 & 148552 \\
\hline (12) & $290.97(71)$ & $7.03(2)^{(9 b)}$ & .030 & .045 & .036 & .972 & .964 & 148540 \\
\hline
\end{tabular}

Note $\chi^{2}=$ chi-square, $R M S E A=$ root mean square error of approximation, $S M S R=$ standardized root mean square residuals, $C F I=$ comparative fit index, $N N F I=$ non-normed fit index, $A I C=$ Akaike information criterion.

$\mathrm{mc}=$ model comparison 
Table 3

Loadings and g-variances for each group (SLD and typically-developing).

\begin{tabular}{|c|c|c|c|c|c|}
\hline \multirow{2}{*}{ Subtests } & \multicolumn{2}{|c|}{ SLD } & \multicolumn{2}{|c|}{ Typical } & \multirow{2}{*}{$\Delta$ Variances } \\
\hline & Load & Var. & Load & Var. & \\
\hline Similarities & .584 & .301 & .621 & .385 & .085 \\
\hline Vocabulary & .569 & .324 & .658 & .434 & .109 \\
\hline Comprehension & .478 & .229 & .522 & .305 & .076 \\
\hline Block Design & .533 & .284 & .554 & .307 & .023 \\
\hline Picture Concepts & .480 & .231 & .604 & .365 & .135 \\
\hline Matrix Reasoning & .626 & .391 & .633 & .401 & .009 \\
\hline Digit Span & .286 & .082 & .483 & .233 & .151 \\
\hline Letter-Number Sequencing & .424 & .180 & .561 & .315 & .136 \\
\hline Coding & .144 & .021 & .281 & .079 & .058 \\
\hline Symbol Search & .301 & .093 & .421 & .177 & .084 \\
\hline
\end{tabular}




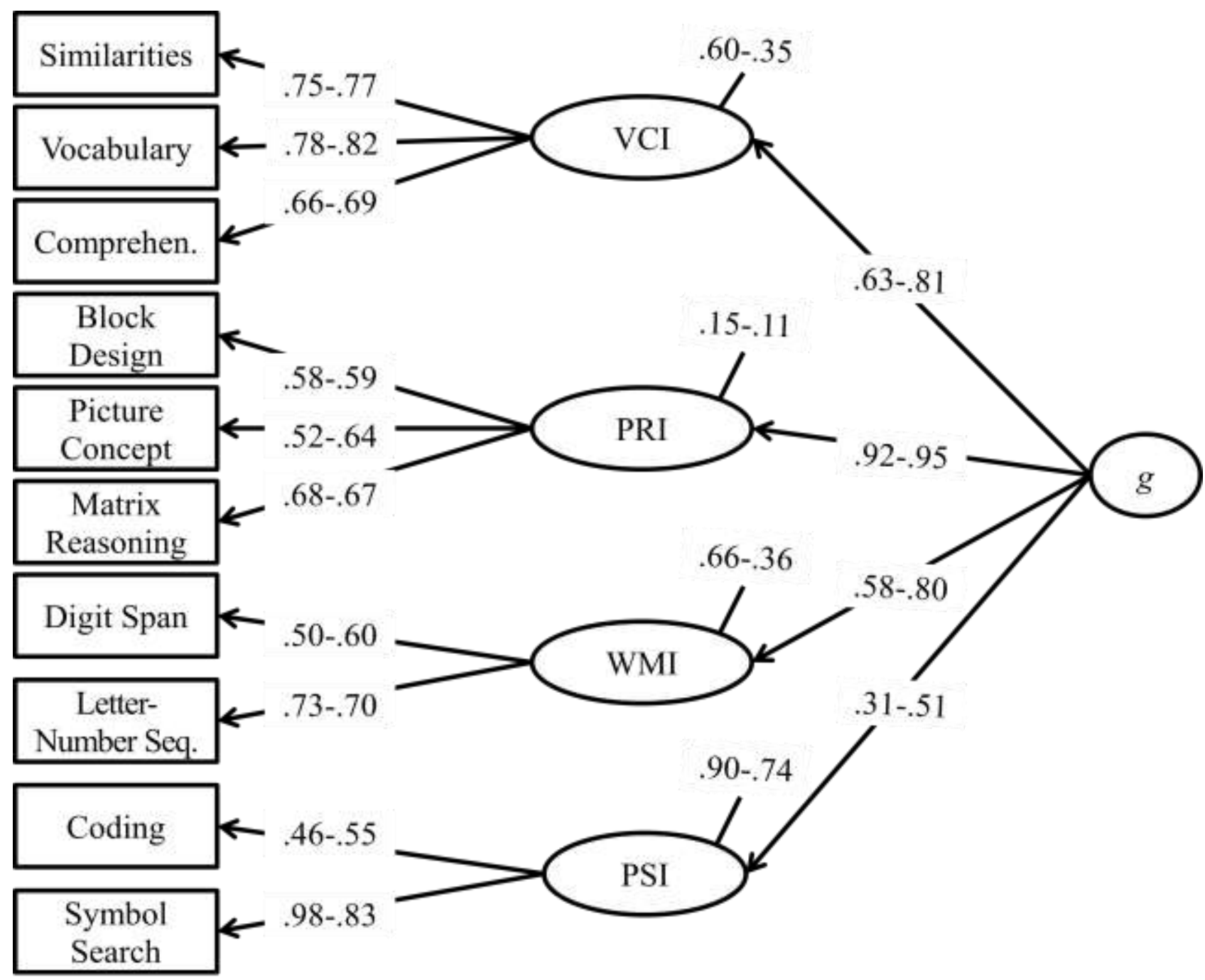

Figure 1. The hierarchical four-factor structure of the WISC-IV in children with SLD (parameter estimates on left) and typically-developing children (parameter estimates on right). $\mathrm{VCI}=$ Verbal Comprehension Index, $\mathrm{PRI}=$ Perceptual Reasoning Index, $\mathrm{WMI}=\mathrm{W}$ orking Memory Index, and PSI = Processing Speed Index. 